\title{
Necessity of Preoperative Computed Tomography in Percutaneous Renal Surgery
}

\author{
Hikmet Topaloglu ${ }^{1}$, Nihat Karakoyunlu ${ }^{2}$, Fuat Aksun ${ }^{3}$, \\ Ugur Ozok ${ }^{4}$,Levent Sagnak ${ }^{5}$ and Hamit Ersoy ${ }^{6}$ \\ 1,2,3,4,5 Deparment of Urology, Dışkapı Yıldırım Beyazıt Training and Research Hospital, Ankara, \\ Turkey \\ ${ }^{6}$ Department of Urology, Hitit University, School of Medicine, Çorum, Turkey
}

Correspondence should be addressed to: Nihat Karakoyunlu; nkarakoyunlu@gmail.com

Received date: 10 June 2014; Accepted date: 23 September 2014; Published date: 22 January 2016

Academic editor: Apul Goel

Copyright (C) 2016. Hikmet Topaloglu, Nihat Karakoyunlu , Fuat Aksun ,Ugur Ozok ,Levent Sagnak and Hamit Ersoy. Distributed under Creative Commons CC-BY 4.0

\begin{abstract}
Aim: Colon injury is an important complication of percutaneous renal surgery. Retrorenal colon on computed tomography (CT) is reported in $10 \%$ of patients in the prone position. Our aim is to discuss the necessity of routine preoperative CT and perform a cost analysis of this procedure. Methods: We retrospectively analyzed the records of 285 patients with renal calculi who underwent percutaneous nephrolithotomy between April 2007 and April 2012. After the $2^{\text {nd }}$ colon perforation, we began to use CT routinely to image stones and to determine the presence of retrorenal colon. We compared the total cost of CTs per incidence of retro colon versus the cost of restoring complications. Results: The first colon injury was extraperitoneal and could be managed conservatively. The cost of this management was only $\$ 280$. The second colon injury was intraperitoneal and could be managed with a series of open surgeries. The cost of these operations was $\$ 8,400$. The cost of diagnosis using CT per case of retrorenal colon was $\$ 1,833$. Conclusion: $C T$, which should be low-dose, if possible, should be performed routinely in all patients to prevent colon injuries. This technique appears to be cost-effective.
\end{abstract}

Keywords: Computed tomography; percutaneous nephrolithotomy; retrorenal colon

\section{Introduction}

Recent advancements in minimally invasive techniques have changed treatment modalities and reduced the use of open surgery. High success rates have been reported with shock wave lithotripsy
(SWL), which is the most minimally invasive process. However, a high stone burden and some calyceal pathologies might reduce the success of this technique while increasing the treatment period (Lingeman et al., 1987). The other minimally invasive method for the

Cite this Article as: Hikmet Topaloglu , Nihat Karakoyunlu , Fuat Aksun ,Ugur Ozok ,Levent Sagnak and Hamit Ersoy (2016)," Necessity of Preoperative Computed Tomography in Percutaneous Renal Surgery ", International Inurnal of Recearrh in Irnloov Vol Jn16 (Jn16) Article In 412365 
treatment of renal stones is percutaneous nephrolithotomy (PNL), which was first reported by Fernström and Johansson in 1976 (Fernström and Johansson, 1976). Colon injury is an important complication of percutaneous renal surgery. Retrorenal colon on computed tomography (CT) is reported in $10 \%$ of patients in the prone position (Figure 1). However, the clinical incidence of colon injury is much lower than this figure would suggest (El-Nahas et al., 2006; Hopper et al., 1987). Today, CT is not used routinely before PNL.

In this retrospective study, we aim to assess the cost-effectiveness of preoperative CT by comparing the total cost of CT per incidence of retrorenal colon to the cost of restoring complications.

\section{Materials and Methods}

We retrospectively analyzed the records of 285 patients with renal calculi who underwent percutaneous nephrolithotomy between April 2007 and April 2012. We excluded patients who had routinely undergone a pre-operative CT for horseshoe kidney, ectopic kidney and renal anomalies and who had previously undergone open renal surgery. Intravenous urography (IVU) was the only imaging method used in the first 123 PNL cases. A second colon perforation occurred in the 123rd patient. We subsequently began to use routine CT to image stones and to determine the presence of retrorenal colon or other abnormalities. Non-contrast whole abdomen CT with a stone protocol was performed in the prone position. All IVU and CT scans were evaluated retrospectively based on digital archives from our hospital.

The authors pre-operatively evaluated the diagnosis of retrorenal colon. During this diagnosis procedure, colon that was present in the percutaneous access tract was evaluated as a retrorenal colon. When calculating the cost of whole abdomen noncontrast helical CT, we used the price list of the Social Security Institute (SGK) of Turkey, which listed a price of 34 US Dollars (\$). The total cost was obtained by multiplying this cost by 162 (i.e., the CT count of patients 123 to 285). During the calculation, we also used the package prices of SGK for the cost of the additional procedures required to correct colon injuries; for the procedures that were not listed, we used the total bill of the hospital to determine the cost. We also calculated the CT scan cost required for one diagnosis of retrorenal colon (i.e., the ratio of the total cost of CT scans to the total number of diagnosed retrorenal cases).

\section{Results}

The first colon injury was extraperitoneal and could be managed conservatively. After this event occurred, a nephrostomy catheter was replaced in the retrorenal area and a double-J catheter was inserted with local anesthesia and fluoroscopic assistance. Oral intake was stopped. Fecal drainage was completed on the $2^{\text {nd }}$ day, oral intake was started on the $7^{\text {th }}$ day, the drain was removed on the $10^{\text {th }}$ day and the patient was discharged on the $12^{\text {th }}$ day. The cost of this management was only $\$ 280$.

The second colon injury was intraperitoneal and could be managed with a series of open surgeries. The treatment was continued in the general surgery clinic. In the first session, a diversion colostomy was performed on the patient, who initially received a double- J catheter. The closure of the colostomy was performed 6 months later as a second session. On the $4^{\text {th }}$ postoperative day, the patient was permitted water intake, on the $5^{\text {th }}$ day, the patient was permitted aqueous food intake, and the on the $7^{\text {th }}$ day, the drain was pulled and the patient was discharged from the hospital. The cost of these operations was $\$ 8,400$. In addition, the authors identified 3 retrorenal colon cases among the 162 total cases; these patients had a high potential risk of injury. In terms of $\mathrm{CT}$, the total cost for the 162 cases was $\$ 5,500$, and the cost of recognizing one case of retrorenal colon was $\$ 1,833$. One of these 3 retrorenal cases was treated with laparoscopy-assisted PNL, and the other two cases were treated with PNL with the guidance of the initial nephrostomy tract that was applied preoperatively during CT. 


\section{Discussion}

PNL is a safe and reliable procedure for the treatment of renal stones. However, PNL is an invasive procedure with reported complication rates of $3 \%$ to $4 \%$ (Michel et al., 2007; de la Rosette et al., 2008). One of the most distressing complications is colonic injury. Although the incidence of this complication is very low (i.e., 0.3 to $1 \%$ of cases) (Skolarikos and de la Rosette, 2008), colonic injury may produce dangerous morbidity in the form of septicemia, peritonitis, abscess formation, and nephrocolic or colocutaneous fistula. The risk factors for colon injury include renal anomalies, such as horseshoe kidney, retrorenal colon, previous intestinal bypass surgery, female sex, elderly, thin patients and colonic distension. The incidence of colonic injury was also greater on the left side, and a more lateral puncture increases the risk (El-Nahas et al., 2006). Nevertheless, no statistically significant evidence has implicated these factors in the development of colonic injury because these reports contained only small numbers of patients or were single case reports. However, we excluded patients with risk factors in this retrospective analysis to focus on the accurate incidence of colon injury in the normal anatomy.

In the literature, retrorenal colon was defined as a retrorenal position of the colon, as determined by a line drawn coronally through the posterior margin of the kidney at any level (Sherman et al., 1985). However, only the presence of colon that intersects the access tract can cause injuries. For example, if a lower pole access was planned and a retrorenal colon was displacing the upper portion of the kidney, this event was not evaluated as a retrorenal colon. For this reason, colonic injuries occur more rarely than retrorenal colon.

Tomographic imaging prior to surgery to evaluate neighboring anatomic structures in the potential puncture path can be a preventive technique. Early diagnosis and treatment are crucial for the management of this injury.
Intraoperatively, the visualization of fecal material during nephroscopy or a bowel opacification with nephrostogram is a sign of colon injury. If this condition is identified intraoperatively, the nephrostomy tube should be replaced into the colon and left as a colonic tube to create a controlled fistula. The urine must be drained with a nephrostomy tube or with a double-J ureteral stent. Broad-spectrum antibiotics must be used. The colonic tube may be removed when intestinal and urinary tract diversion have been proven (Korkes et al., 2009).

Postoperatively, fecal material in the nephrostomy tube, blood in the feces, pneumaturia, nausea, ileus, vomiting, leukocytosis, fever, sepsis or peritonitis should be associated with bowel injury (Zuckerman and Desai, 2011). In the case of extraperitoneal injuries, conservative management may be preferred. The urinary and intestinal tracts must be drained separately via a collecting system with a double-J ureteral stent or a nephrostomy tube. If sepsis or peritonitis is suspected, open surgical repair must be performed.

The identification of a retrorenal colon is of utmost importance for the prevention of colonic injury during the establishment of a percutaneous tract. Mousavi-Bahar et al. reported 671 cases and demonstrated that mortality occurred in only one patient; death was associated with colonic injury (Mousavi-Bahar et al., 2011). In our series, the first colon injury was extraperitoneal and could be managed conservatively. The cost of this management was only $\$ 280$. However, the second colon injury was intraperitoneal and could be managed with a series of open surgeries. The cost of these operations was $\$ 8,400$. Preoperative abdominal CT can determine the relationship between the colon and the kidney. Tuttle et al. studied 83 patients with supine and prone CT scans and found that that the colon was located along the expected path of the lower pole access tract in $15 \%$ of prone scans compared to only $6 \%$ of supine scans (Tuttle et al., 2005). Regardless, the incidence of visceral injury is low in clinical series of both prone and 
supine PNL. El-Nahas' review of 5,039 prone PNL procedures found a colonic injury rate of only $0.3 \%$ (El-Nahas et al., 2006). The largest supine series to date included more than 550 patients, with no visceral injuries reported (Valdivia Uría et al., 1998).

In the literature, due to the low incidence of retrorenal colon and the high cost and radiation exposure that are associated with CT, CT is not recommended for all patients before PNL (Korkes et al., 2009). However, preoperative CT has been recommended for patients with a high risk of retrorenal colon, as discussed above. In the past, we preferred using IVU for diagnosing patients. The high dose of radiation associated with CT discouraged us from using this technique. Until the $123^{\text {rd }}$ case in the presented series, we did not use CT, but after colon perforation in the $123^{\text {rd }}$ case, we realized that the advantages of CT outweighed the disadvantages of this procedure. Today, we always visualize stones with CT. We believe that CT should be used prior to performing PNL for two reasons: first, to detect the presence of retrorenal colon, and second, to plan the most suitable access route. CT-planned access has been reported to increase the success rate of PNL (Chalasani et al., 2010; Thiruchelvam et al., 2005). In our series (285 patients), the cost of recognizing one retrorenal colon case using CT was $\$ 1,833$, but because colon injury is a serious complication with a high risk of morbidity and a risk of mortality, an optimal diagnosis should be achieved to avoid this complication.

However, some factors that constrain the implications of our findings should be emphasized. The retrospective nature of the study is a degree of limitation. In addition, the extended study period causes a time effect, with differences and heterogeneity in the study material caused by constant developments in instruments and stone disintegration methods.

Although the use of CT is a contentious issue due to high-level radiation, we believe that CT should always be performed before PNL to identify the presence of retrorenal colon and to avoid colon injury. CT can also reveal the stone location and provide information that is helpful for accessing the kidney (Ghani et al., 2009). During CT, one must be careful to minimize the radiation dose. White et al. reported that low-dose CT could be performed safely during pregnancy (White et al., 2007); therefore, we believe that all patients should be evaluated using lowdose CT before PNL.

As the incidence of colon injury is between $0.3 \%$ and $1 \%$, the number of CTs required to prevent one instance of this complication could be calculated to be between 100 and 333. Because the cost of a CT in our medical system is 34 US Dollars, the total cost for detecting 1 retrorenal colon case might be lower than the cost in other countries. Thus, rationally, the costs should be calculated based on the conditions of the country in question. However, CT is beneficial not only in terms of detecting colon injuries but also in terms of preventing other complications and determining the structure of the stone. We recommend that despite the low cost of surgery, routine CT should be evaluated in terms of cost and performed to prevent complications that could result in a colostomy and require a series of operations.

Conflict of Interest Note: No conflicts of interests exist.

\section{References}

1. Chalasani, V., Bissoon, D., Bhuvanagir, A.K., Mizzi, A., Dunn, I.B., 2010. Can. J. Urol. 17, 5082-5086.

2. El-Nahas, A.R., Shokeir, A.A., El-Assmy, A.M., Shoma, A.M., Eraky, I., El-Kenawy, M.R., El-Kappany, H.A., 2006. Urology 67, 937-941.

3. Fernström, I., Johansson, B., 1976. Scand. J. Urol. Nephrol. 10, 257-259.

4. Ghani, K.R., Patel, U., Anson, K., 2009. J. Endourol. Endourol. Soc. 23, 1633-1639. 
5. Hopper, K.D., Sherman, J.L., Williams, M.D., Ghaed, N., 1987. Invest. Radiol. 22, 298-302.

6. Korkes, F., Lopes Neto, A.C., Lucio, J., 2nd, Bezerra, C.A., Wroklawski, E.R., 2009. J. Endourol. Endourol. Soc. 23, 569-573.

7. Lingeman, J.E., Coury, T.A., Newman, D.M., Kahnoski, R.J., Mertz, J.H., Mosbaugh, P.G., Steele, R.E., Woods, J.R., 1987. J. Urol. 138, 485-490.

8. Michel, M.S., Trojan, L., Rassweiler, J.J., 2007. Eur. Urol. 51, 899-906; discussion 906.

9. Mousavi-Bahar, S.H., Mehrabi, S., Moslemi, M.K., 2011. Urol. J. 8, 271-276.

10.De la Rosette, J.J.M.C.H., Zuazu, J.R., Tsakiris, P., Elsakka, A.M., Zudaire, J.J., Laguna, M.P., de Reijke, T.M., 2008. J. Urol. 180, 2489-2493.

11.Sherman, J.L., Hopper, K.D., Greene, A.J., Johns, T.T., 1985. J. Comput. Assist. Tomogr. 9, 339-341.
12.Skolarikos, A., de la Rosette, J., 2008. Curr. Opin. Urol. 18, 229-234.

13.Thiruchelvam, N., Mostafid, H., Ubhayakar, G., 2005. BJU Int. 95, 12801284.

14.Tuttle, D.N., Yeh, B.M., Meng, M.V., Breiman, R.S., Stoller, M.L., Coakley, F.V., 2005. J. Vasc. Interv. Radiol. JVIR 16, 14891492.

15.Valdivia Uría, J.G., Valle Gerhold, J., López López, J.A., Villarroya Rodriguez, S., Ambroj Navarro, C., Ramirez Fabián, M., Rodriguez Bazalo, J.M., Sánchez Elipe, M.A., 1998. J. Urol. 160, 1975-1978.

16.White, W.M., Zite, N.B., Gash, J., Waters, W.B., Thompson, W., Klein, F.A., 2007. J. Endourol. Endourol. Soc. 21, 1255-1260.

17.Zuckerman, D.A., Desai, A., 2011. Semin. Interv. Radiol. 28, 438-443. 